\title{
THORACIC EPIDURAL ANALGESIA IMPROVES LUNG FUNCTION IN CABG PATIENTS
}

Paul K. Tenenbein MD, Doug Maguire MD, Roland Debrouwere MD, Peter C. Duke MD and Stephen E. Kowalski MD.

Department of Anesthesia, Health Sciences Centre, University of Manitoba, GH611 - 820

Sherbrook Street, Winnipeg, Manitoba, R3A 1R9

\section{INTRODUCTION}

Pulmonary complications commonly occur following Coronary Artery Bypass Graft (CABG) surgery increasing morbidity and mortality. High thoracic epidural analgesia (TEA) decreases pulmonary complications and provides superior analgesia in thoracic surgery patients. We hypothesize that TEA will improve pulmonary function and provide superior analgesia in patients undergoing CABG surgery.

\section{METHODS}

Ethics board approval was obtained, and this prospective, randomized, controlled trial was conducted. Consecutive patients undergoing CABG surgery were randomized into 2 groups. Preoperatively, patients in the epidural group received a thoracic epidural and intraoperatively, $0.75 \%$ ropivacaine was infused followed postoperatively with a $0.2 \%$ ropivicaine infusion. The control group received PCA morphine. Both groups received standardized general anesthetics. Outcome measurements included visual analog pain scores, spirometry data and atelectasis scores. Statistical analysis using SAS version 8.2 was conducted adhering to the intention to treat principle. Statistical tests included 2-sample t-tests for parametric data and 2-sample Wilcoxon and Chi square tests for non-parametric data. Repeated measures ANOVA followed by least squares means were used to compare multiple groups. Data is presented as the mean \pm standard deviation.

\section{RESULTS}

Twenty-five patients were enrolled in each group. The demographics of the groups were similar. The epidural group required more phenylephrine intraoperatively $(4.26 \pm 2.78 \mathrm{mg}$ vs. $2.06 \pm 1.75 \mathrm{mg} \mathrm{p}<0.001)$. Time to extubation was similar in the epidural and control groups $(15.3 \pm 37.6 \mathrm{~min}$ vs. $10.3 \pm 12.6 \mathrm{~min}, \mathrm{p}=0.26)$. Patients in the epidural group had significantly less pain on the operative day and for the next 2 days. Compared to baseline, the FEV1 was significantly higher in the epidural group on the first and second postoperative days $(43.7 \pm 12.2 \%$ vs. $36.4 \pm 12.0 \%, \mathrm{p}<0.002$ and $43.3 \pm 12.5 \%$ vs. $38.4 \pm 11.0 \%, \mathrm{p}<0.05)$. There was significantly more atelectasis in the control group 4 hours postoperatively $(\mathrm{p}<$ 0.04). There were no complications related to epidural hematoma and no permanent neurologic sequalae.

\section{DISCUSSION}

High thoracic epidural analgesia decreases postoperative pain, improves pulmonary function and decreases atelectasis. Our results support the use of thoracic epidural analgesia in patients undergoing CABG surgery. 